\title{
TEORÍA EFECTIVA PARA ESTRELLAS BINARIAS Y SU APLICACIÓN A ONDAS GRAVITACIONALES
}

\author{
EFFECTIVE THEORY FOR BINARY STARS AND ITS \\ APPLICATION TO GRAVITATIONAL WAVES
}

\begin{abstract}
Anthonny Freddy Canazas Garay ${ }^{1}$
RESUMEN

Las recientes detecciones de ondas gravitacionales han traído mayor atención al problema de obtener predicciones de alta precisión para los espectros de radiación gravitacional emitidos por sistemas binarios constituidos por agujeros negros o estrellas de neutrones. Este trabajo explica como la teoría de campos efectiva aplicada a la gravitación puede ser usada para describir objetos extendidos interactuando gravitacionalmente como en el caso de un sistema binario. Usando métodos que usualmente se aplican en teoría cuántica de campos se muestra como el sistema binario emite ondas gravitacionales.
\end{abstract}

Palabras clave. - Ondas gravitacionales, Teoría de campos efectiva, Relatividad general, astrofísica, Agujero negro, Estrella de neutrones

\begin{abstract}
The recent detections of gravitational waves have brought renewed attention to the problem of obtaining high accuracy predictions for the gravitational radiation spectra emitted by binary systems with black holes or neutron stars constituents. This work explains how effective field theory applied to gravitation can be used to describe a gravitationally interacting extended object as in the binary system case. Gravitational wave emission from the binary system is shown by using methods that are usually applied in quantum field theory.
\end{abstract}

Key words.- Gravitational waves, Effective field theory, General relativity, Astrophysics, Black hole, Neutron star.

\section{INTRODUCCIÓN}

El presente trabajo es producto de cálculos y discusiones efectuadas durante la visita del autor al ICTP-SAIFR con motivo del evento "School on Effective Field Theory across Length Scales" [1] y está basado en parte en las lecciones [2] de ondas gravitacionales de Rafael Porto en dicho evento. Los métodos de la Teoría de Campos Efectiva (TCE) permiten dar cuenta de los efectos que surgen en las diferentes escalas del sistema binario de forma análoga a las TCEs construidas para estudiar las diferentes escalas en física de altas energías o materia condensada. Esta línea de investigación cobra fuerza en vista de las recientes detecciones de ondas gravitacionales realizadas por la colaboración científica LIGO [3y 4].

La TCE se usa si se quiere tener una descripción aproximada en cierta escala de una teoría en particular.

La Revista Científica TECNIA protege los derechos de autor bajo la Licencia 4.0 de Creative Commons: Attribution 4.0 International (CC B Y 4.0) ${ }^{1}$ Doctor, docente de la Facultad de Ciencias de la Universidad Nacional de Ingeniería. 
Es decir, se desea calcular los efectos de la física de distancias cortas sobre la física a una escala de interés sin usar la teoría completa, ya sea porque ésta no es conocida o porque es conocida pero fuertemente acoplada. Esta física a distancias cortas suela llamarse "física UV" la cual esta descrita en términos de grados de libertad UV o grados de libertad pesados. La física de largas distancias en la escala de interés es descrita por grados de libertad livianos o modos livianos. La idea clave que hace posible las TCEs es que dada una teoría de campos descrita por una acción $S[\phi, \Phi]$ con grados de libertad livianos $\phi$ y algunos campos pesados $\Phi$ se puede integrar fuera los modos $\Phi$ en la integral por caminos:

$$
e^{i S_{\text {eff }}[\phi]}=\int D \Phi(x) e^{i S[\phi, \Phi]}
$$

obteniéndose una acción efectiva describiendo las interacciones de los campos livianos entre ellos. Resulta que en general, $S_{\text {eff }}[\phi]$ puede expresarse como:

$S_{e f f}[\phi]=\sum_{i} c_{i} \int O_{i}(x) d^{4} x$

los coeficientes $c_{\bar{i}}$ son usualmente llamados "coeficientes de Wilson". Si las simetrías que sobreviven a bajas energías son conocidas, entonces los $O_{i}(x)$ deben ser términos respetando las simetrías. En resumen, para construir una TCE se necesitan básicamente dos pasos:

-Identificar los grados de libertad a la escala de interés.

-Construir el Lagrangiano más general para estos grados de libertad que sea consistente con las simetrías.

Se usarán unidades en las que la constante de Planck $\hbar$ y la velocidad de la luz $c$ son iguales a la unidad. Además se trabajará con la masa de Planck reducida $M_{P l}=\left(32 \pi G_{N}\right)^{-1 / 2}$, donde $G_{N}$ es la constante de gravitación de Newton.

\section{BINARIA DE OBJETOS COMPACTOS}

La binaria de interés está formada por dos objetos compactos orbitando mutuamente alrededor de su centro de masa. Estos pueden ser agujeros negros o estrellas de neutrones.

Se tiene una escala típica para el tamaño de los objetos, la cual está dada por el radio de Schwarzschild:

$R_{S}=\frac{2 G_{N} m}{c^{2}}$,

donde $m$ es la masa característica de los objetos, y también la escala de separación $r$ como se aprecia en la Figura 1.

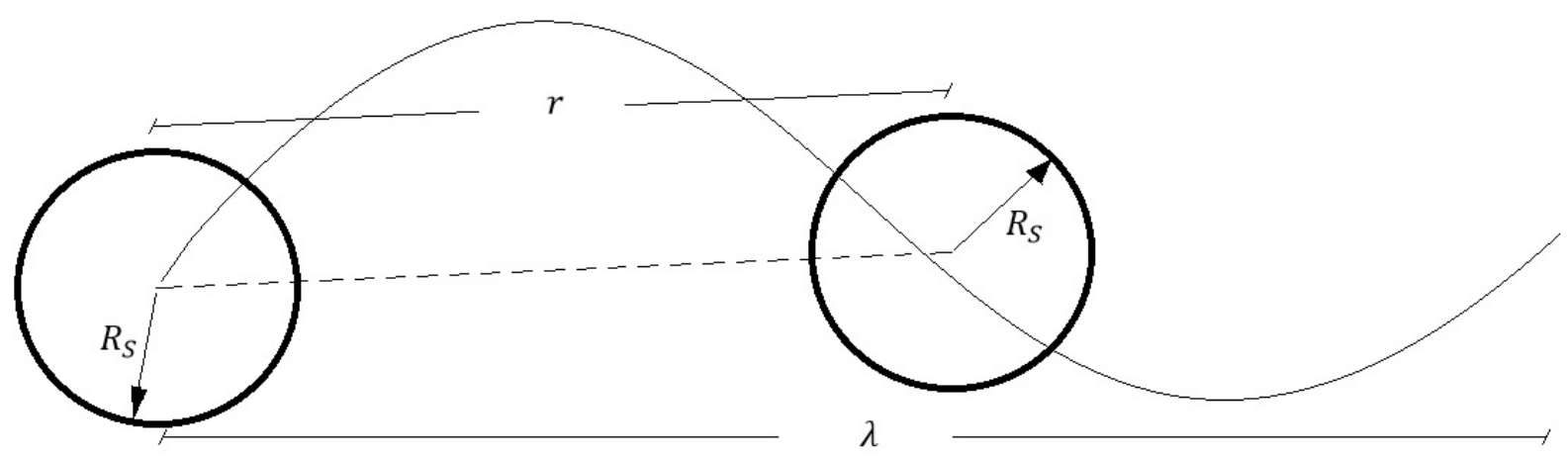

Fig. 1 Objetos compactos orbitando. Se muestra esquemáticamente las diferentes escalas de longitud involucradas. 
Además para poder encontrar un parámetro de expansión se necesita que la distancia de separación sea más grande que los objetos, es decir, el régimen de validez es:

$R_{S} \ll r$.

Lo importante para hacer uso de la TCE es encontrar un parámetro de expansión. Si los objetos se mueven lentamente, con velocidad relativa $v$, de modo que el teorema del virial para un estado ligado resulta:

$$
\frac{v^{2}}{2} \sim \frac{G_{N} m}{r}
$$

entonces se tiene también que $v^{2} / c^{2} \sim R_{S} / r$. Por tanto, siempre que $R_{S} / r \ll 1$ se sabrá que la órbita será no relativista. Se tiene entonces una consistencia en las expansiones de parámetros $R_{S} / r$ y $v / c$. A partir de aquí se usará unidades en las que $c=1$. Cuando la separación es más grande que el tamaño se tiene entonces:

$\frac{R_{S}}{r} \sim v^{2} \ll 1$

Además hay una longitud de onda de radiación típica. Los objetos al estarse moviendo, como se verá más adelante, adquieren un momento cuadrupolar que está cambiando con el tiempo de modo que la situación es similar a un dipolo o antena emitiendo ondas electromagnéticas. Por tanto esta longitud de onda (o frecuencia si se quiere) es $\lambda=r / v$ la cual cumple también $\lambda \gg r \mathrm{y}$ se tiene por tanto

$\frac{r}{\lambda} \sim v \ll 1$

Ese es el parámetro de expansión y esa es la forma en la que se construye una teoría efectiva y lo relevante en TCE es que no importa cuál es la física UV. Solo importan las simetrías de la física a largas distancias y cuáles son los grados de libertad livianos que están entrando a tallar y en el presente caso será una TCE de gravedad. No solo para el caso de la binaria sino también para cualquier objeto extendido en el fondo de un campo gravitacional de onda larga el sistema físico será descrito por una serie de términos en una acción efectiva. Estos términos modifican la física UV y tienen un conteo de potencias que indica que en alguna escala o distancia esta teoría se viene abajo, pero mientras se permanece dentro del régimen de validez de la teoría todo estará bien. El conteo de potencias dará factores de $R_{S} / r$ o $r / \lambda$, en ambos casos resultan potencias en la velocidad. Esta expansión en potencias de $v$ es la llamada expansión Post-Newtoniana, el n-ésimo orden en esta expansión es del orden de

$\mathrm{nPN} \sim v^{2 \mathrm{n}}$.

Si hay simetría de inversión del tiempo, solo habrá potencias pares de $v$. Es importante tener siempre presente que aunque se está haciendo una expansión en velocidades, lo que realmente se está haciendo es una expansión en el cociente de las escalas. Esta TCE es genérica y describe a diversos sistemas, pero los coeficientes de Wilson en la teoría serán diferentes en cada caso.

En Relatividad General completa se usaría la acción de Einstein-Hilbert $S_{E H}\left[g_{\mu v}\right]$ para la métrica más un término que representa la interacción de la materia con la métrica. Este término se puede modelar como una fuente con tensor de energíamomentum $T^{\mu v}$

$S_{E H}\left[g_{\mu v}\right]+S_{M}=-2 M_{P l}^{2} \int R \sqrt{-g} d^{4} x-\frac{1}{2 M_{P l}} \int T^{\mu v} h_{\mu v} d^{4} x$

donde $R=g^{\mu v} R_{\mu \nu}$ es el escalar de Ricci que se construye a partir del tensor de Ricci $R_{\mu v}$.

Resolver analíticamente es muy complicado, por eso la Relatividad General Numérica se ha convertido en una importante área de investigación desde hace décadas. Uno se puede preguntar cuál es el sentido de estudiar a la binaria analíticamente si es que existen simulaciones numéricas. La razón de este esfuerzo es debido a que resolver analíticamente siempre ayuda a desarrollar nuevas 
técnicas, usar técnicas ya conocidas en ámbitos nuevos o descubrir aspectos que no se podrían visualizar con resultados puramente numéricos, además de poder escanear parámetros. Cuando se hace una simulación se introducen ciertas masas, espines y si se quiere crear una plantilla se debe hacer muchas simulaciones. Pero esta información justamente requiere ser extraída de los datos observacionales y para esto se necesita escanear diferentes posibles parámetros, formas de onda o plantillas y para eso se requiere tener un cierto control analítico.

Más aún, cuando se miden las frecuencias en la banda LIGO, entre $10 \mathrm{~Hz}$ y $1 \mathrm{kHz}$ aproximadamente, realmente se están observando solo los últimos ciclos de la binaria antes de que los agujeros negros se fusionen y terminen convirtiéndose en un agujero negro de Kerr. La señal GW150914 detectada por LIGO duró unas dos décimas de segundo observándose ocho ciclos mientras que la señal GW151226 duró alrededor de un segundo observándose unos 55 ciclos. Para el caso de dos estrellas de neutrones orbitando, que aún no se han detectado, se tiene un estimado de unos pocos minutos de observación. A pesar de ser solo unos minutos, las velocidades serían enormes y el número de ciclos llegaría a $10^{5}$. Incluso una pequeña desviación entre los cálculos teóricos de las formas de onda y los datos observacionales se verá amplificada a lo largo del gran número de ciclos.

Con Relatividad General Numérica no se puede simular más de 100 y solo en ciertos casos simples, algunas simulaciones pueden tardar meses. Definitivamente es algo que no se puede usar para obtener información precisa sobre las masas o espines de los objetos o eventualmente sobre cuáles son los grados de libertad UV.

Un intento para obtener soluciones analíticas es desdoblar la métrica en

$g_{\mu v}=\eta_{\mu v}+h_{\mu v} / M_{p l}$
Insertando esto en la acción de Einstein-Hilbert esquemáticamente:

$S_{E H}=\int h \partial^{2} h+\frac{1}{M_{P l}} h h \partial \partial h+\cdots$

se obtiene la expansión Post-Minkowskiana. Esto ocurre en el sector de una partícula, no se ha puesto aún al segundo objeto. Luego será necesario lidiar con las otras escalas de la binaria. Una manera conveniente de hacer cálculos sistemáticos es construir TCEs que capturen la física relevante en cada escala separadamente.

\section{ACCIÓN EFECTIVA DE LÍNEA DE UNIVERSO}

Se tiene una clara separación: el tamaño del objeto, la separación y la onda gravitacional. Pero al final lo que se quiere es escribir una teoría para un objeto extendido, cualquiera, interactuando con un campo gravitacional de onda larga. Cada una de las componentes en la binaria está interactuando con dos campos gravitatorios: la energía potencial gravitatoria y la emisión de ondas gravitacionales que produce un fondo donde la binaria se mueve. Lo que se debe hacer primero es ignorar la radiación. Se pueden distinguir, en general, dos tipos de modos gravitacionales: modos de distancia corta y modos de distancia larga. A distancias del orden $r$ se tiene un modo de potencial que está asociado a un potencial cuasi instantáneo proporcional a $1 / r$ como en la electrodinámica. Es un modo off-shell ya que en el espacio de Fourier solo tiene las componentes espaciales como se verá en la siguiente sección. El otro campo, la emisión de ondas gravitacionales, tiene un mayor alcance.

Es esta misma teoría la que se usará luego para ver al sistema binario completo como un objeto extendido interactuando con un campo gravitacional de onda larga que será, en este caso, la emisión de onda gravitacional. Lo resaltante es que los grados de libertad cortos que serán integrados fuera no son solo los que están en la escala de $R_{S}$ sino también los que están en la escala $r$. Es decir, se empieza con un objeto interactuando 
con un grado de libertad externo el cual para la escala $R_{S}$ es distancia larga, pero para la escala $r$ es distancia corta.

Separando grados de libertad cortos $g_{\mu \nu}^{S}$ y grados de libertad largos $g_{\mu \nu}^{L}$ se tiene para la métrica:

$g_{\mu v}=g_{\mu v}^{S}+g_{\mu \nu}^{L}$

Hay que integrar fuera estos $g_{\mu \nu}^{S}$ en la integral por caminos. La acción $S_{M}$ se convierte en un término $S_{\text {int }}\left[x^{\alpha}, g_{\mu v}^{S}\right]$ describiendo las posiciones de los constituyentes de la estrella $\delta x^{\alpha}=x^{\alpha}-x_{C M}$ y su geometría. Integrando fuera estos grados de libertad geométricos se tiene para la aproximación de punto de ensilladura:

$e^{i S_{E H}\left[g_{\mu v}^{L}\right]+i S_{e f f}^{p p}\left[x C M g_{\mu v}^{L}\right]}=\int D \delta x^{\alpha} D g_{\mu v}^{S} e^{i S_{E H}\left[g_{\mu v}\right]+i S_{\text {int }}\left[x^{\alpha}, g_{\mu v}^{S}\right]}$

Siempre se llega a esta descripción de una partícula puntual interactuando con un campo gravitacional de onda larga, es decir, la acción $S_{\text {eff }}^{p p}$ es una integral sobre la línea de universo. Ahora se escribe una serie de grados de libertad livianos y las simetrías que la teoría debe respetar. Uno de estos grados de libertad es el campo métrico $g_{\mu v}^{L}$. La estrella puede trasladarse y rotar libremente y es justamente por eso que el grado de libertad $x_{C M}$ se mantiene, además de una base o tétrada que proporciona los grados de libertad de rotación que, en aras de la simplicidad, no se tomarán en cuenta en la discusión. Las simetrías son los difeomorfismos de la Relatividad General y la invariancia de re-parametrización de la línea de universo. Luego lo que se hará esencialmente es una expansión multipolar al escribir todos los términos que respeten estas simetrías. El primer término que se escribe es la masa (el monopolo), $-m \int d \tau$, usando el tiempo propio. En principio se puede añadir términos proporcionales a $R$ y a $R_{\mu \nu}$, las simetrías de la teoría lo permiten y se puede hacer el conteo de potencias sin dificultad. Usando coeficientes de Wilson $C_{R}$ y $C_{V}$ se tiene:

$$
S_{\text {eff }}^{p p}=\int\left(-m+C_{R} R+C_{V} R_{\mu v} \dot{x}^{\mu} \dot{x}^{v}\right) d \tau
$$

donde los puntos denotan derivación temporal. Sin embargo estos últimos dos términos causan el siguiente problema si el objeto de interés es esféricamente simétrico: al calcular la función de 1punto para obtener la métrica de Schwarzschild un término lineal en $g_{\mu \nu}^{L}$ de la expansión de $R$ hará que aparezca el coeficiente $C_{R}$ yendo en contra del teorema de Birkhoff.

Lo bueno es que estos términos pueden anularse si se hace una redefinición del campo $g_{\mu \nu}^{L}$ salvo un término de contacto proporcional a una delta. Éste es similar al término de contacto que aparece en las correcciones relativistas del átomo de Hidrógeno. Como no hay superposición ese término no importa, pero cuánticamente sí serían importantes y habría correcciones cuánticas al teorema de Birkhoff. Por tanto, los términos $R$ y $R_{\mu \nu}$ pueden ser eliminados de la teoría salvo contra-términos.

Ahora se puede escribir invariantes a partir del tensor de Riemann $R_{\mu \alpha v \beta}$. Por comodidad se usará el tensor de Weyl $C_{\mu \alpha v \beta}$ que es la parte sin traza del tensor de Riemann. Con este tensor se puede hacer el mismo truco que en el electromagnetismo de descomponer en partes eléctricas y magnéticas:

$$
E_{\mu v}=C_{\mu \alpha v \beta} \dot{x}^{\alpha} \dot{x}^{\beta}, \quad B_{\mu v}=\frac{1}{2} \epsilon_{\mu \alpha \beta \sigma} C_{v \rho}^{\alpha \beta} \dot{x}^{\sigma^{x^{\rho}}}
$$

Las cuales obedecen $g_{\alpha \beta} E^{\alpha \beta}=g_{\alpha \beta} B^{\alpha \beta}=0 \mathrm{y}$ $E_{\alpha \beta} \dot{x}^{\beta}=B_{\alpha \beta} \dot{x}^{\beta}=0$. Con esto lo único que queda por hacer es

$S_{\text {eff }}^{p p}=\int\left(-m+C_{E} E_{\mu v} E^{\mu v}+C_{B} B_{\mu v} B^{\mu v}\right) d \tau$

Los términos son cuadráticos en la métrica, por lo que no se genera ningún problema. Esto es todo lo necesario para el caso estático. Pero si además se 
quiere estudiar la emisión de ondas, en vez de esos términos se debe escribir:

$$
S_{\text {eff }}^{p p}=\int\left(-m+Q_{i j}^{E} E^{i j}+Q_{i j}^{B} B^{i j}\right) d \tau
$$

Aquí los $Q_{i j}^{E(B)}$ son los cuadrupolos eléctricos (magnéticos) sin traza. Se usa solo índices espaciales ya que se está usando la tétrada en la cual $e_{0}^{\mu}=\dot{x}^{\mu}$ y las componentes espaciales forman un sistema localmente plano de forma que $E_{A B}=e_{A}^{\mu} e_{B}^{v} E_{\mu v}$ y similarmente para $B_{A B}$. En esta base $E_{0 A}=B_{0 A}=0$ por lo que solo las componentes espaciales $E_{i j}$ son relevantes. Se puede escribir realmente todo esto en términos de $\operatorname{los} E_{\mu v}$, pero se quiere las tres componentes usuales de un cuadrupolo. Esto se hace en analogía al término de energía $\vec{P} \cdot \vec{E}$ en el caso del electromagnetismo en el cuál la susceptibilidad es el factor de proporcionalidad $\vec{P}=\chi \vec{E}$ y lo que se obtiene es algo proporcional a $E^{2}$. Con esto ya se puede describir fenómenos más interesantes como la emisión de ondas gravitacionales. El objeto en cuestión no tiene un cuadrupolo dependiente del tiempo. Pero si el objeto tuviera esto es lo que se debe que escribir. Ahora, ¿cómo se recupera el caso de simetría esférica de aquí? Extendiendo la idea de la susceptibilidad en el electromagnetismo al caso de la gravedad. Esto significa que el $Q_{i j}^{E}$ es proporcional a $E_{i j}$ y este factor de proporcionalidad es el coeficiente $C_{E}$, despreciando los términos con derivadas. Como $E^{\mu \nu}$ es ortogonal a $e_{0}^{\mu}$ se puede ver que $E_{i j} E^{i j}$ es lo mismo que $E_{\mu \nu} E^{\mu \nu}$.

Este $Q_{i j}^{E}$ sería lo que suele llamarse la "respuesta", pero en general hay dos contribuciones a los momentos cuadrupolares o multipolares (en general):

$Q_{i-i_{n}}^{E(B)}=\left\langle Q_{i-i_{n}}^{E(B)}\right)+\left(Q_{i-i_{n}}^{E(B)}\right)_{R}$
Las contribuciones de distancia corta en ausencia de perturbaciones externas están incorporadas en el valor de expectación $\left\langle Q_{\tilde{i}-\tilde{i}_{n}}^{E(B)}\right)$, mientras que la respuesta $\left(Q_{\tilde{i}-\tilde{l}_{n}}^{E(B)}\right)_{R}$ representa el multipolo inducido por presencia de un campo externo. Trabajando en el espacio de frecuencias la relación entre $\left(Q_{i j}^{E}\right)_{R}$ y $E_{i j}$ se convierte en una función de frecuencias $\omega$ llamada función de respuesta $F(\omega)$ la cual se puede expandir en serie de potencias. Estas frecuencias en el dominio del tiempo significan derivar respecto al tiempo. Usando la simetría de inversión en el tiempo $(\omega \rightarrow-\omega)$ de la parte real de esta respuesta:

$\operatorname{Re} F(\omega)=C_{E}+C_{\tilde{E}}\left(R_{S} \omega\right)^{2}+\mathcal{O}\left(\left(R_{S} \omega\right)^{4}\right)$

Similarmente se puede hacer esto para el octupolo y así sucesivamente. Esta serie de funciones de respuesta de multipolos dará todos los términos posibles que se pueden agregar en la acción efectiva.

Ahora, la escala de los coeficientes es una cuestión importante si se quiere saber hasta qué punto de la expansión Post-Newtoniana se necesita ir para poder distinguir un agujero negro de una estrella de neutrones. Obviamente si el objeto en cuestión tiene unas 30 masas solares se trata de un agujero negro, pero si se tiene algo del orden de una masa solar, no se sabe. Esencialmente lo que se hace es dispersión de ondas gravitacionales por un objeto extendido. Por simplicidad se considera un agujero negro de modo que no hay parámetros describiendo la estructura interna, solamente el radio $R_{S}$. La sección eficaz de dispersión depende de la frecuencia $\omega$ del gravitón y se calcula en la teoría efectiva y en Relatividad General completa.

La amplitud, en la teoría efectiva, para un gravitón que se dispersa en el campo de un agujero negro es:

$i \mathcal{A} \sim \cdots+i \frac{C_{E(B)}}{M_{P l}^{2}} \omega^{4}+\cdots$ 
Habrá varios términos más que contribuyan como por ejemplo el término producido por $-m \int d \tau$ en la acción (2), pero lo importante es que se puede aislar el término deseado de la parte que es analítica en $\omega$ la cual se puede expandir. Esta es una importante lección: los modos livianos producen términos no analíticos y más vale retenerlas en la teoría efectiva para que se cancelen con los términos no analíticos de la teoría completa. De otro modo cuando se haga la comparación se obtienen grandes logaritmos y el resultado diverge. Por tanto lo único que queda es algo analítico en $\omega$ que se puede expandir y por lo que la sección eficaz debe tener un término:

$\sigma_{T C E} \sim \cdots+\frac{C_{E(B)}{ }^{2}}{M_{P l}{ }^{4}} \omega^{8}+\cdots$

En la teoría completa de Relatividad General el cálculo contiene un solo parámetro por lo que se espera obtener:

$\sigma_{G R}^{B H}=R_{S}^{2} f\left(R_{S} \omega\right)$

donde $f(z)$ es una función analítica. Si se expande en potencias de $R_{S} \omega \ll 1$ se tiene:

$\sigma_{G R}^{B H} \sim R_{S}^{2}\left(\cdots+\left(R_{S} \omega\right)^{8}+\cdots\right)$

Comparando las expresiones $\sigma_{T C E}$ y $\sigma_{G R}^{B H}$ (salvo factores numéricos) se tiene:

$C_{E(B)} \sim M_{P l}{ }^{2} R_{S}{ }^{5}$

Estos coeficientes $C_{E}$ y $C_{B}$ son las susceptibilidades del objeto, es decir, cuanto se deforma al colocarlo en un campo externo. La Relatividad General clásica indica que estos coeficientes son cero para el agujero negro como ha sido demostrado por varios grupos [5] calculando la función de respuesta. Por el contrario, para estrellas de neutrones se tiene un valor finito para $C_{E(B)}$. El coeficiente $C_{B}$ entrará a un orden más alto en la expansión Post-Newtoniana por lo que la forma de distinguir un agujero negro de una estrella de neutrones es por el coeficiente $C_{E}$.

\section{EMISIÓN DE ONDA GRAVITACIONAL}

Del término $h \partial^{2} h$ en (1) se obtiene la función de Green como una integral en el espacio de momentum:

$G(x, y)=\frac{1}{(2 \pi)^{4}} \int \frac{e^{-i k(x-y)}}{k_{0}^{2}-k^{2}} d^{4} k$.

Con la que la solución de las ecuaciones de Einstein para una fuente con tensor $T_{\mu \nu}$ está dada por:

$h_{\mu v}(x)=\int G(x, y) T_{\mu v}(y) d^{4} y$

Si se trata de una fuente localizada y estática entonces no hay nada que dependa del tiempo salvo $e^{-i k_{0}\left(x^{0}-y^{0}\right)}$ en $G(x, y)$. La integral en el tiempo $y^{0}$ dará una distribución $\delta\left(k_{0}\right)$ la cual elimina el $k_{0}$ y deja la parte estática $\boldsymbol{k}$, la cual tiene una escala de $1 / r$. Mientras que la fuente permanezca estática la región de la función de Green que importa es donde $k_{0}=0$, pero si se va moviendo lentamente hay velocidades y ya no se forma la delta. Sin embargo se puede realizar el siguiente truco para hacer el cálculo como derivadas de deltas: se expande en $k_{0} /|\boldsymbol{k}|$ y luego el $k_{0}$ sube:

$\frac{1}{k_{0}^{2}-\boldsymbol{k}^{2}}=-\frac{1}{\boldsymbol{k}^{2}}\left(1+\frac{k_{0}^{2}}{\boldsymbol{k}^{2}}+\cdots\right)$

lo que producirá dos derivadas debido a $k_{0}^{2}$ luego de integrar en $k_{0}$. Se obtendrán deltas, pero con derivadas temporales. De este modo se puede calcular, por ejemplo, las correcciones perturbativas en $k_{0}$ al potencial estático. Esto esencialmente significa que la escala de $k_{0} \sim v / r$ es mucho menor que $\mid \boldsymbol{k} \|$ para la contribución cuasi estática la que es llamada modo de potencial. Por otro lado, los modos de radiación están on-shell, con momentum del orden $v / r$, y estos son los responsables de la radiación que se propaga hasta el detector. Es así 
como se introduce la idea de separar en modos de radiación y modos de potencial.

Se tiene por tanto:

$$
\left(k_{0}, \boldsymbol{k}\right)_{p o t} \sim\left(\frac{v}{r}, \frac{1}{r}\right), \quad\left(k_{0}, \boldsymbol{k}\right)_{r a d^{\sim}}\left(\frac{v}{r}, \frac{v}{r}\right)
$$

Ahora se separarán los modos de potencial $H_{\mu V} \mathrm{y}$ modos de radiación $\bar{h}_{\mu v}$ en la métrica para obtener una teoría efectiva de onda larga:

$$
g_{\mu \nu}=\bar{g}_{\mu \nu}+H_{\mu v} / M_{P l} \quad \bar{g}_{\mu \nu}=\eta_{\mu \nu}+\bar{h}_{\mu \nu} / M_{P l}
$$

La acción efectiva para la teoría de radiación se obtiene por tanto de:

$$
e^{i S_{E H}\left[\bar{g}_{\mu v}\right]+i S_{E f f}^{r a d}\left[x_{n} \bar{h}_{\mu V}\right]}=\int D H_{\mu V} e^{i S_{E H}\left[g_{\mu V}\right]+i S_{E f f}^{p p}\left[x, g_{\mu v}\right]}
$$

Usando las simetrías de la teoría a largas distancias se obtiene:

$S_{e f f}^{r a d}\left[\boldsymbol{x}, \bar{h}_{\mu v}\right]=\int\left[-M+Q_{i j}^{E} E^{i j}+Q_{i j}^{B} B^{i j}+\cdots\right] d \tau$

donde $M$ representa la masa del sistema binario el cual es reemplazado, por tanto, por un objeto puntual dotado de una serie de momentos multipolares. Además el centro de masa se ubica en reposo en el origen de coordenadas de modo que $\sqrt{\bar{g}_{00}} d t=d \tau$. Nótese que para el caso de la binaria la respuesta es muy pequeña al contrario del caso del agujero negro o estrella de neutrones individual en la que la respuesta es importante. Para la binaria lo que importa es el valor de expectación ya que ésta es la parte que va a producir ondas gravitacionales. Los $Q_{i j}^{E(B)}$ no solo tienen los cuadrupolos asociados al movimiento del par de objetos sino también la energía potencial que también está radiando. Es decir, los $Q_{i j}^{E(B)}$ son renormalizados por la energía de unión.

Ahora se calculará la potencia total radiada en ondas gravitacionales y la forma de onda a una distancia grande comparada con el tamaño de la binaria. Aunque los observables que se desea calcular son clásicos, se puede usar los métodos de TECNIA 25 (2) 2015 la teoría cuántica de campos para extraer de $S_{\text {eff }}^{\text {rad }}\left[\boldsymbol{x}, \bar{h}_{\mu \nu}\right]$ estos observables. La potencia total radiada $d E / d t$ se relaciona con la tasa de emisión de gravitones $\Gamma_{h}(\boldsymbol{k})$ mediante:

$$
\frac{d E}{d t}=\sum_{h} \int k^{0} d \Gamma_{h}(\boldsymbol{k})
$$

Además:

$$
\begin{gathered}
d \Gamma_{h}(\boldsymbol{k})=\frac{1}{T} \frac{d^{3} \boldsymbol{k}}{(2 \pi)^{2} 2|\boldsymbol{k}|}\left[\mathcal{A}_{h}(\boldsymbol{k})\right]^{2} \\
\frac{i}{4 M_{P l}} \epsilon_{i j}^{*}(\boldsymbol{k}, h) \omega^{2}
\end{gathered}
$$

Fig. 2 Diagrama de Feynman producido por el término $Q^{i j} E_{i j}$ en la acción efectiva de radiación.

Se calcula la amplitud correspondiente al acople con $Q_{i j}^{E}$ cuyo diagrama se muestra en la Figura 2. Los términos de multipolos superiores se están despreciando así como también el cuadrupolo $Q_{i j}^{B}$, por lo que en adelante se obviará el superíndice $E$. La amplitud resulta:

$$
i_{c} \mathcal{A}_{h}(\boldsymbol{k})=\frac{i}{4 M_{p l}} \epsilon_{i j}^{*}(\boldsymbol{k}, h) \omega^{2} Q^{i j}(\omega)
$$

Reemplazando en la potencia (3):

$\frac{d E}{d t}=\frac{G_{N}}{\pi T} \int_{0}^{\infty}\left[\frac{\omega^{6}}{5}\left|Q^{i j}(\omega)\right|^{2}\right] d \omega$

Pasando al dominio del tiempo se obtiene la conocida fórmula de radiación del cuadrupolo:

$\left.\frac{d E}{d t}=\frac{G_{N}}{5} \mid\left(\frac{d^{3}}{d t^{3}} Q^{i j}(t)\right)^{2}\right)$

Similarmente, usando la función de 1-punto con el propagador retardado, se puede calcular la forma de onda $\bar{h}_{i j}$ : 
$\bar{h}_{i j}=\frac{1}{4 M_{P l}^{2}} \int D_{i j k l}^{r e t}\left(t-t^{\prime}, x\right) \ddot{Q}^{k l}\left(t^{\prime}\right) d t^{\prime}$

Dónde:

$D_{i j k l}^{r e t}\left(t-t^{\prime}, \boldsymbol{x}\right)=-\theta\left(t-t^{\prime}\right) \frac{\delta\left(t-t^{\prime}-\| \boldsymbol{x} \mid\right)}{4 \pi|\boldsymbol{x}|} P_{i j k l}$

con lo que se obtiene:

$\bar{h}_{i j}=-\frac{2 G_{N}}{|\boldsymbol{x}|} \ddot{Q}_{i j}(t-\| \boldsymbol{x} \mid)$

el cuál es el resultado esperado.

\section{CONCLUSIONES}

Se ha mostrado como los métodos de la TCE pueden ser exitosamente aplicados al problema de la binaria encontrándose un rango de escalas de longitud físicamente importantes, desde el tamaño de los constituyentes hasta la longitud de onda de la radiación gravitacional. La simetría de inversión del tiempo es importante para esto facilitando algunos cálculos. De este modo la TCE ha probado ser una herramienta poderosa, quedando pendiente la inclusión del espín, como en [6], lo cual será crucial para construir plantillas precisas de formas de onda para la inminente era de la "astronomía de ondas gravitacionales".

La contribución del coeficiente $C_{E}$ a la forma de onda lleva los efectos de los grados de libertad a la escala $R_{S}$ para un objeto extendido. Sería útil, por tanto, obtener analíticamente expresiones precisas de la fase de la onda gravitacional con la dependencia en $C_{E}$ y verificar modelos de la ecuación de estado para estrellas de neutrones.

Ya que este coeficiente es diferente en el caso de agujeros negros, significa que se deforman diferente. El hecho que $\operatorname{los} C_{E}$ se anulen para los agujeros negros va en contra de lo esperado ya que no hay ninguna simetría que proteja a $C_{E}$ de recibir correcciones posiblemente grandes. Es un problema de ajuste fino desde el punto de vista de la TCE el hecho que $C_{E}$ se anule. Esto se vuelve menos misterioso cuando se toma en cuenta la naturaleza singular del agujero negro. ¿Qué significa deformarse para un agujero negro? ¿Cómo se distingue entre la deformación del agujero negro de la deformación del espacio-tiempo en sí?

Por otro lado, la detección de un $C_{E}$ no nulo para el agujero negro indicaría la existencia de nueva física a esa escala y podría dilucidar aspectos de gravedad cuántica. En ese sentido diversas propuestas se han formulado para resolver la paradoja de la información y esta podría ser una oportunidad de poner a prueba estas ideas y en última instancia descubrir los grados de libertad del agujero negro responsables de tan peculiares aspectos.

\section{REFERENCIAS}

1. http://www.ictp-saifr.org/?page_id=9163

2. Porto, R. A., "The effective field theorist's approach to gravitational dynamics", Phys.Rept. 633 (2016) 1-104.

3. Abbott, B. P. et al. "Observation of gravitational waves from a binary black hole merger", Phys.Rev.Lett. 116 (2016) no.6, 061102.

4. Abbott, B. P. et al. "GW151226: Observation of gravitational waves from a 22-solar-mass binary black hole coalescence", Phys.Rev.Lett. 116 (2016) no.24, 241103.

5. Chakrabarti, S., Delsate, Tr., Steinhoff, J., "New perspectives on neutron star and black hole spectroscopy and dynamic tides", arXiv:1304.2228 [gr-qc]

6. Porto, R. A., Ross, A., Rothstein, I. Z., "Spin induced multipole moments for the gravitational wave amplitude from binary inspirals to 2.5 Post-Newtonian order", JCAP 1209 (2012) 028.

Correspondencia: acanazasg@uni.edu.pe 